\title{
Estratificación socio-espacial y por ingresos en los servicios de desarrollo infantil y cuidado en la Ciudad de Buenos Aires ${ }^{1}$
}

\section{Socio-spatial and economic stratification in Early Childhood Education and Care services in the City of Buenos Aires}

\author{
Gabriela L. Marzonetto \\ Centro Interdisciplinario para el Estudio de las Políticas Publicas, CIEPP \\ gmarzonetto@ciepp.org.ar
}

\begin{abstract}
RESUMEN
En sociedades desiguales, las diferencias de acceso y calidad de los servicios educativos y de cuidado que prestan los establecimientos de desarrollo infantil condicionan las oportunidades de los niños de familias de menores ingresos. Considerando que Argentina no es ajena a este fenómeno, en este artículo se analiza si el modo en que se organiza socialmente el cuidado de niños y niñas en la Ciudad de Buenos Aires opera como mecanismo de estratificación social. Para responder este interrogante se parte de una estrategia metodológica que combina estadística descriptiva -test de diferencia de medias y comparación simple de medias de ingresos per cápita familiares por comuna y tipo de establecimiento de desarrollo infantil al que asisten los niños- e inferencial, mediante la aplicación de un modelo de regresión logística (modelo logit) que permita establecer significancia estadística en la correlación entre la asistencia a establecimientos de desarrollo infantil y los ingresos familiares y ponderar la probabilidad de concurrencia a estos establecimientos según el nivel de ingreso familiar.
\end{abstract}

Este artículo pretende contribuir al debate sobre la estratificación y segmentación social presente en los servicios públicos, como los educativos y de desarrollo infantil, en la región.

\section{PALABRAS CLAVE}

Servicios de desarrollo infantil, Organización social del cuidado, estratificación social.

\begin{abstract}
In unequal societies, differences in access and quality of early child education and care services determine children's opportunities. Considering that Argentina is not alien to this phenomenon, this article asks if the way in which child care is socially organized in the City of Buenos Aires operates as a mechanism of social stratification. To answer this question the article parts of a methodological strategy that combines descriptive statistics - means tests and simple comparison of means using per capita income per family and type of early child education and care services that children attend- and inferential statistic, through the application of a logistic regression model that allows to establish statistical significance in the correlation between attendance to early child education and care services and family income, and to ponderate the probability of attendance to these establishments according to the family income level.
\end{abstract}

Overall, this article aims to contribute to the discussion about social stratification and segmentation prevalent in public services, such as child education and care services, in the region.

\section{KEYWORDS}

Early childhood education and care services, social organization of childcare, social stratification.

1 Agradezco los valiosos comentarios y aportes de Julio Aguirre, Rubén Lo Vuolo y Julia Pérez Zorrilla, así como los de los revisores anónimos de este trabajo. 
GAPP. Nueva Época - N. 18, noviembre 2017 - ISSN: 1989-8991 - DOI: 10.24965/gapp.v0i18.10464 - [Págs. 43-61]

Estratificación socio-espacial y por ingresos en los servicios de desarrollo infantil y cuidado en la Ciudad de Buenos Aires

Gabriela L. Marzonetto

\begin{abstract}
SUMARIO
1. INTRODUCCIÓN. 2. ORGANIZACIÓN SOCIAL DEL CUIDADO, SERVICIOS DE DESARROLLO INFANTIL Y ESTRATIFICACIÓN SOCIAL. 3. METODOLOGÍA. 4. RESULTADOS DE INVESTIGACIÓN. 4.1. LA ORGANIZACIÓN SOCIAL DEL CUIDADO DE NIÑOS PEQUEÑOS EN LA CIUDAD DE BUENOS AIRES. OFERTA DE SERVICIOS DE DESARROLLO INFANTIL Y CUIDADO. 4.2. ANÁLISIS DE MEDIAS DE INGRESOS PER CÁPITA FAMILIARES DE LAS FAMILIAS CON NIÑOS QUE ASISTEN A ESTABLECIMIENTOS DE DESARROLLO INFANTIL. 4.3. MODELO LOGIT SOBRE EL IMPACTO DEL NIVEL DE INGRESOS PER CÁPITA FAMILIAR EN LA PROBABILIDAD DE ASISTENCIA A ESTABLECIMIENTOS DE DESARROLLO INFANTIL. 4.3.1. Modelo logit. 5. DISCUSIÓN. 6. REFERENCIAS BIBLIOGRÁFICAS.
\end{abstract}

\title{
1. INTRODUCCIÓN
}

La primera infancia constituye un momento importante para el desarrollo cognitivo y social de los niños y las niñas, por lo que las oportunidades que se brinden en esta primera etapa constituida desde el nacimiento hasta los 5 años de edad resulta crucial tanto para su escolaridad futura como para sus oportunidades en la vida adulta. En sociedades desiguales estas actividades, tendientes al cuidado y la educación, esenciales para el desarrollo infantil -junto con otros factores-, condicionan las oportunidades de los niños de familias de menores ingresos, cuestión que repercute sobre la estratificación social y la reproducción intergeneracional de la pobreza.

Estudios de psicología experimental y de neurociencia sostienen que las bases cognitivas decisivas quedan fijadas en el curso de la primera infancia (Barnett, 2013; UNICEF, 2014), por lo que el aprendizaje que ha tenido lugar en la etapa preescolar es fundamental para la motivación y las capacidades de aprendizaje del niño al momento de ingresar a la escuela.

Esto demuestra que los orígenes sociales marcan profundamente las oportunidades y es en este punto donde se hace evidente la necesidad de preocuparnos por políticas públicas que se enfoquen en la niñez desde la primera infancia, y no solo a partir de la etapa escolar.

Numerosos estudios han demostrado los efectos positivos a corto, mediano y largo plazo de los servicios educativos pre-escolares en niños y niñas de bajos recursos ${ }^{2}$. Asimismo, estos reparan en la importancia de los entornos familiares de los niños y el nivel educativo de ambos padres, más allá del nivel de ingresos familiares (Felfe et al., 2012; Addabbo y Di Tommaso, 2014).

Para que estos servicios públicos puedan derivar en resultados positivos para los niños y niñas ${ }^{3}$, es importante que sean de alta calidad, entendiendo por esto a servicios que cumplan contenidos educativos mínimos, regulen la cantidad de niños por sala y exijan competencias mínimas en los profesionales a cargo de los mismos. Si los servicios que se proveen tienen carencias en materia de calidad, no importa cuánto se avance en la extensión de los mismos, ya que las diferencias de calidad pueden derivar en consecuencias negativas en el desarrollo cognitivo de los niños de padres con menor nivel educativo y bajo nivel de ingresos (Felfe et al., 2012).

Con todo, hay una correlación directa entre los ingresos de los padres, la asistencia escolar de sus hijos y los ingresos de estos en el futuro, lo que coadyuva a la reproducción intergeneracional de la pobreza. En otras palabras, sin una inversión social en primera infancia «la herencia social sale reforzada» (EspingAndersen y Palier, 2011: 63). Podemos sostener entonces que las políticas de desarrollo infantil y cuidado en la primera infancia tienen un efecto relevante en las oportunidades de vida a futuro y la reproducción de la desigualdad y la estratificación social.

Esta situación no es ajena en la Ciudad Autónoma de Buenos Aires (en adelante CABA), donde la baja cantidad de cupos en los establecimientos de desarrollo infantil del sistema educativo público hace que, por un lado los niños de familias de ingresos medios y altos concurran a establecimientos de gestión privada; y por otro lado que los niños de menores ingresos vean retrasada la participación en estos establecimientos hasta la edad de escolaridad obligatoria, o en el caso de acceder lo hagan en establecimientos que brindan servicios de tipo asistencial orientados principalmente a niños en situación de vulnerabilidad social donde los contenidos educativos y pedagógicos ocupan un lugar marginal. A esta situación se le suma que en la CABA se presentan diferencias en el nivel de asistencia a espacios de cuidado por edad, por tipo de servicio

2 Ver al respecto: ADDABBO y DI TOMMASO, 2014; BARNETT, 2013; FELFE, et al., 2012.

3 El estudio de FELFE, et al.(2012) demuestra que el efecto positivo de los servicios de cuidado se ve también en la participación de las mujeres en el mercado de empleo. 
(educativo-asistencial) y por tipo de gestión (pública-privada) de acuerdo a la comuna de residencia. Demostrando esto que la estratificación social, también se observa por localidad geográfica.

En este trabajo se busca, por un lado, responder si el modo en que se organiza socialmente el cuidado de niños y niñas en la CABA opera como mecanismo de estratificación social y por otro lado, generar evidencia que permita abonar los debates académicos sobre la estratificación social y segmentación de servicios públicos en la región. A tal fin, en el siguiente apartado se presenta el debate sobre el problema de la organización social del cuidado, los servicios de desarrollo infantil y la estratificación social, luego se realiza un diagnóstico de la actual forma de organización social del cuidado de niños pequeños en la CABA en base a información referida a la oferta de servicios a los que estos acceden, y por último se busca responder a la pregunta de investigación a través del tratamiento de análisis estadísticos descriptivo e inferencial.

\section{ORGANIZACIÓN SOCIAL DEL CUIDADO, SERVICIOS DE DESARROLLO INFANTIL Y ESTRATIFICACIÓN SOCIAL}

El 'cuidado' puede ser entendido como «una actividad vital para el bienestar de la población y como parte esencial de una organización social y política en la que intervienen, además de sujetos individuales, instituciones públicas y privadas» (Faur 2014,14). Es decir, el cuidado involucra todas las actividades que las personas realizamos para satisfacer nuestras necesidades básicas. A pesar de esto, la caracterización de estas actividades como actividades de cuidado, y de éste último como problemática social, es un fenómeno relativamente nuevo y viene aparejado a la entrada de las mujeres al mundo del trabajo remunerado fuera del hogar. A su vez, en el marco de sociedades desiguales, las tareas de cuidado por lo general son realizadas por las mujeres por lo que la forma en que se organiza la provisión del cuidado en una sociedad tiene importantes repercusiones en términos de la igualdad de género (Esquivel, et. al., 2012; Razavi, 2007; Rodriguez Enríquez y Pautassi, 2014).

El problema del cuidado de personas y el modo en que éste se organiza en las sociedades ha sido estudiado principalmente por la economía feminista (England, Budig y Folbre, 2002), prestando atención al valor económico del trabajo reproductivo no remunerado que se realiza en los hogares así como a los costos que implica su invisibilización, considerando para ello el impacto del cuidado en la participación laboral femenina y el uso del tiempo que dedican varones y mujeres a las actividades productivas y reproductivas (Esquivel, 2009, 2012; Rodríguez Enríquez, 2015).

Los orígenes del término 'cuidado' se remontan a la conceptualización de la condición femenina, para derivar más recientemente hacia el análisis del Estado de Bienestar y de la institucionalidad que gobierna la organización sectorial del cuidado y del tipo de políticas subyacentes (Daly y Lewis, 2000 en Sojo, 2011: 22). Budlender (2008), plantea que el trabajo de cuidado no remunerado es un área de estudio que ha sido invisibilizada por los economistas y otros especialistas en desarrollo. No obstante, éste es realizado por diferentes actores sociales (en su mayoría mujeres) que realizan diferentes tareas tanto remuneradas como no remuneradas, que tienen significativas implicancias tanto en el bienestar de las personas como en el crecimiento económico, ya que las tareas de cuidado son las que permiten la reproducción de la fuerza de trabajo (Batthyány, 2015; Staab y Gerhard, 2011).

Los análisis del cuidado desde el estudio de los Estados y Regímenes de bienestar, hacen hincapié en los diferentes pilares de bienestar que participan en lo que las académicas feministas llaman la Organización Social del Cuidado (en adelante OSC). La OSC refiere a la distribución de las responsabilidades y tareas de cuidado y de los tiempos dedicados a las mismas, entre los hogares, el Estado, los mercados y las organizaciones comunitarias por un lado, y entre varones y mujeres, por el otro (Rodriguez Enríquez y Pautassi, 2014). Estos estudios demuestran que con contadas excepciones, los riesgos asociados al cuidado siguen concentrados en las familias y la preocupación explícita de los gobiernos es incipiente y heterogénea (Sojo, 2011). A su vez, las políticas de infancia que afectan al cuidado de niños y niñas se han situado progresivamente dentro de un marco de derechos, pero discurren por los cauces tradicionales, carentes de un marco de referencia común en la temática del cuidado (2011: 6).

Siguiendo lo anterior, se considera que el Estado ha jugado tradicionalmente un rol menor en lo que respecta a las tareas de cuidado de niños pequeños en comparación con otras áreas del bienestar social. Debido a ello, para entender el modo en el que se organiza socialmente el cuidado debemos ir más allá del «triángulo de bienestar» integrado por el Estado, el Mercado y las Familias (Esping-Andersen, 2008) y 
agregar a este un componente asociado a los arreglos informales/comunitarios y al trabajo no remunerado y pensar al cuidado en términos de un «diamante de bienestar» (Razavi, 2007, 2011)4.

La literatura consultada considera que la forma en que se organiza socialmente el cuidado de niños pequeños en la Argentina es inequitativa debido a que las responsabilidades de cuidado se encuentran desigualmente distribuidas en tres niveles distintos. En primer lugar, a nivel macro, hay una desigual distribución de las responsabilidades de cuidado entre los cuatro componentes del diamante de cuidado, por lo que el mismo se encuentra muy segmentado. En segundo lugar, a nivel de las familias, la desigualdad en la distribución de responsabilidades se verifica también entre varones y mujeres (Rodriguez Enríquez y Pautassi, 2014; Pautassi y Zibecchi, 2010). En tercer lugar, a nivel individual las desigualdades se profundizan en relación al tipo de servicio al que acceden los niños y niñas dependiendo tanto del nivel de ingresos familiares como del lugar de residencia.

Las particularidades del ordenamiento entre el Estado, el mercado, las familias y la comunidad, genera niveles distintos de desmercantilización y desfamiliarización, que a su vez afectan las relaciones sociales. La desmercantilización se produce cuando el Estado presta un servicio entendiéndolo como un asunto de derecho, y por ello el acceso al mismo es independiente de la participación de las personas en el mercado (Esping-Andersen, 1993: 42-43). Por su lado, la desfamiliarización se asocia a la medida en la que el acceso al bienestar social se desvincula de los arreglos y responsabilidades familiares. En otras palabras, la desfamiliarización reduce la dependencia individual de la familia y maximiza el control sobre los recursos económicos de las personas «independientemente de las reciprocidades familiares o conyugales» (Sunkel, 2006: 3).

Por ello entendemos que las políticas sociales, en particular las de desarrollo infantil y cuidado operan como sistemas de estratificación social (Esping-Andersen, 1993: 33). Esto se debe a que el modo en el que se combinan distintos mecanismos de acceso a distintos tipos de beneficios, estableciendo beneficiarios (insiders) y no beneficiarios (outsiders), sumado a la "generosidad» de las prestaciones y los mecanismos de financiación de las mismas, generan efectos relevantes tanto en la distribución del ingreso como de las oportunidades de vida. Por ello, los «rasgos organizativos del bienestar ayudan a determinar la articulación de la solidaridad social, las divisiones de clase y la diferenciación del status»(1993: 81).

En este sentido, se entiende por estratificación social a la división vertical y jerárquica de los distintos estratos sociales (Pakulski, 2006: 586). Debido a ello el análisis de la estratificación social implica mucho más que el estudio del estatus ocupacional, implica también el análisis de las relaciones de género, de raza, el acceso a la educación y el modo de organización del trabajo no remunerado, entre otras. En este sentido, la estratificación social es un fenómeno multidimensional abordado por varios enfoques teóricos y tradiciones metodológicas (Ritzer y Michael 2011: 619-624). Aquí nos ocuparemos sólo de la relación entre la estratificación social y la OSC.

Siguiendo esta línea argumental, el análisis sobre las políticas de desarrollo infantil y cuidado debe atender al modo en que estas afectan los patrones de la organización social del cuidado, ya que la OSC responde al modo en que el Estado regula las relaciones sociales a través de los procesos de (des)mercantilización y (des)familiarización, y éstos a su vez nos permiten observar en qué medida se reproducen mecanismos de estratificación social. En otras palabras, en la medida en que las políticas de cuidado y desarrollo infantil logran, a la vez, desmercantilizar y desfamiliarizar el cuidado de niños y niñas -reduciendo así la segmentación de los servicios- estas tienen un efecto directo sobre la estratificación social.

\section{METODOLOGÍA}

Luego de la definición de las categorías analíticas, en este trabajo se busca responder a la pregunta de investigación a partir de una estrategia metodológica cuantitativa que combina información estadística de tipo descriptiva y análisis inferencial. La primera parte de la estrategia de tipo descriptiva se centra en la reconstrucción del panorama actual de la organización social del cuidado y de servicios de desarrollo infantil en la CABA en base al análisis de información estadística proveniente del Censo Nacional de Vivienda y Población de 2010 del Instituto Nacional de Estadísticas y Censos (INDEC), de la Encuesta Anual de Hogares 2013 del Gobierno de la CABA (GCBA), de los anuarios educativos del Ministerio de Educación de la Ciudad

4 El concepto de diamante de bienestar agrega al sector comunitario y no gubernamental al esquema tripartito Estado-mercadofamilia. De este modo el esquema para analizar la producción de bienestar se amplia y permite incorporar a actores tales como: organizaciones comunitarias, Iglesias, ONGs, entre otros. 
y del Ministerio de Educación de la Nación, y de la Encuesta Nacional de Condiciones de Vida de la Niñez y Adolescencia (ECOVNA) realizada por el Ministerio de Desarrollo Social de la Nación y UNICEF en 2012.

La segunda parte de la estrategia metodológica combina estadística descriptiva e inferencial a fin de poner a prueba la hipótesis de que los niños de familias de menores ingresos asisten menos a establecimientos de desarrollo infantil que los niños de familias de mayores ingresos. Para ello se recurre en un primer momento a un análisis de 'comparación de medias' y un análisis de 'test de diferencia de medias' de ingresos per cápita familiares (IPCF) de las familias con niños que asisten a establecimientos de desarrollo infantil, por comuna y por diferencias entre servicios públicos y privados, para observar la relación entre los ingresos familiares y el tipo de servicio al que los niños asisten. En un segundo momento se realiza un 'modelo Logit' a fin de establecer la significancia estadística en la correlación entre la asistencia a establecimientos de desarrollo infantil y los ingresos familiares y ponderar la probabilidad de concurrencia a estos establecimientos según el nivel de ingreso familiar. A partir de este modelo se calcula la probabilidad de asistencia a establecimientos de desarrollo infantil por nivel de ingresos familiares, para de ese modo completar el análisis estadístico y la puesta a prueba de la hipótesis.

\section{RESULTADOS DE INVESTIGACIÓN}

\subsection{La organización social del cuidado de niños pequeños en la Ciudad de Buenos Aires}

Para analizar las necesidades de cuidado y las estrategias de las familias para resolverlo, debemos tomar en consideración el estrato socio-económico de éstas. La situación socioeconómica de los hogares afecta las estrategias familiares de cuidado debido a que las familias -y dentro de estas las mujeres- realizan elecciones sobre cómo resolver las tareas de cuidado condicionadas por un contexto de bases materiales y normativas diversas (Faur, 2014: 57).

Esto se agrava cuando observamos que la capacidad de desfamiliarizar el cuidado está asociada a la posibilidad de trasladar las responsabilidades de cuidado desde las familias hacia otras instituciones sociales (Esquivel, et al. 2012: 33), ya sean instituciones públicas que permitan que el Estado asuma parte de esta tarea, o la posibilidad de resolverlas por la vía del mercado (ya sea en la contratación de servicio doméstico o de jardines y salas maternales de gestión privada). Esta última opción, no obstante, sólo es posible en familias con elevado poder adquisitivo por lo que la persistencia de la misma se asocia con los mecanismos de estratificación por ingresos.

\section{GRÁFICO 1. DIVISIÓN GEOGRÁFICA DE LA CABA POR COMUNAS}

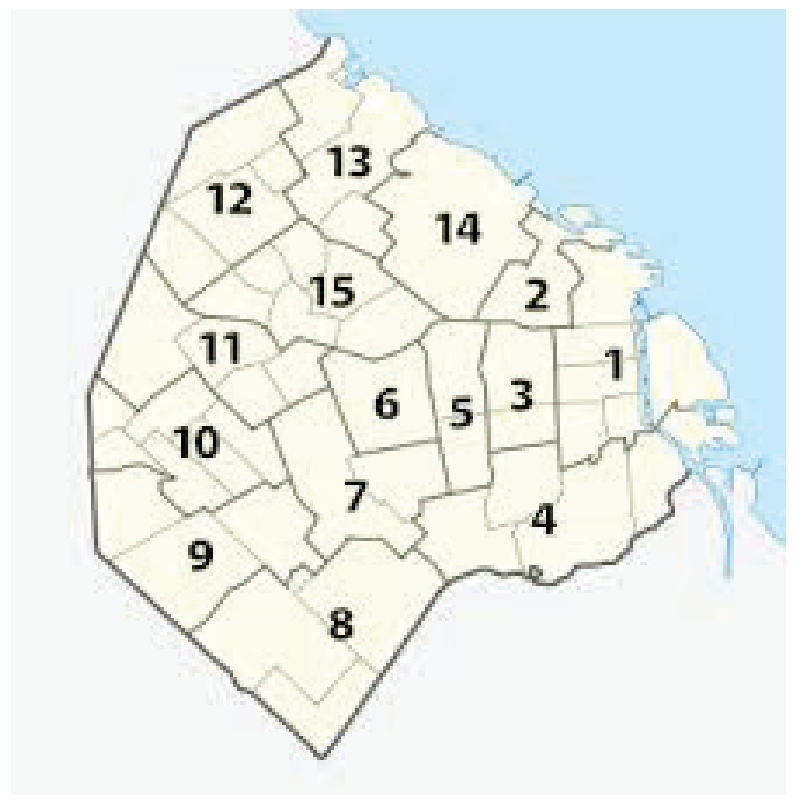

Fuente: Dirección general de estadísticas y censos (Ministerio de Hacienda CABA) 2012 
Frente a esto, es importante destacar que en la CABA encontramos variaciones en relación a la situación económica de los hogares, la composición de los mismos y la comuna de residencia (ver gráficos 1 y $2)^{5}$. Esto es indicativo de la estratificación socio-espacial existente en la Ciudad, cuestión que, como se verá en la siguiente sección, también impacta en el acceso a servicios de cuidado y desarrollo infantil.

\section{GRÁFICO 2. MEDIA DE INGRESOS PER CÁPITA FAMILIAR POR COMUNA ${ }^{6}$}

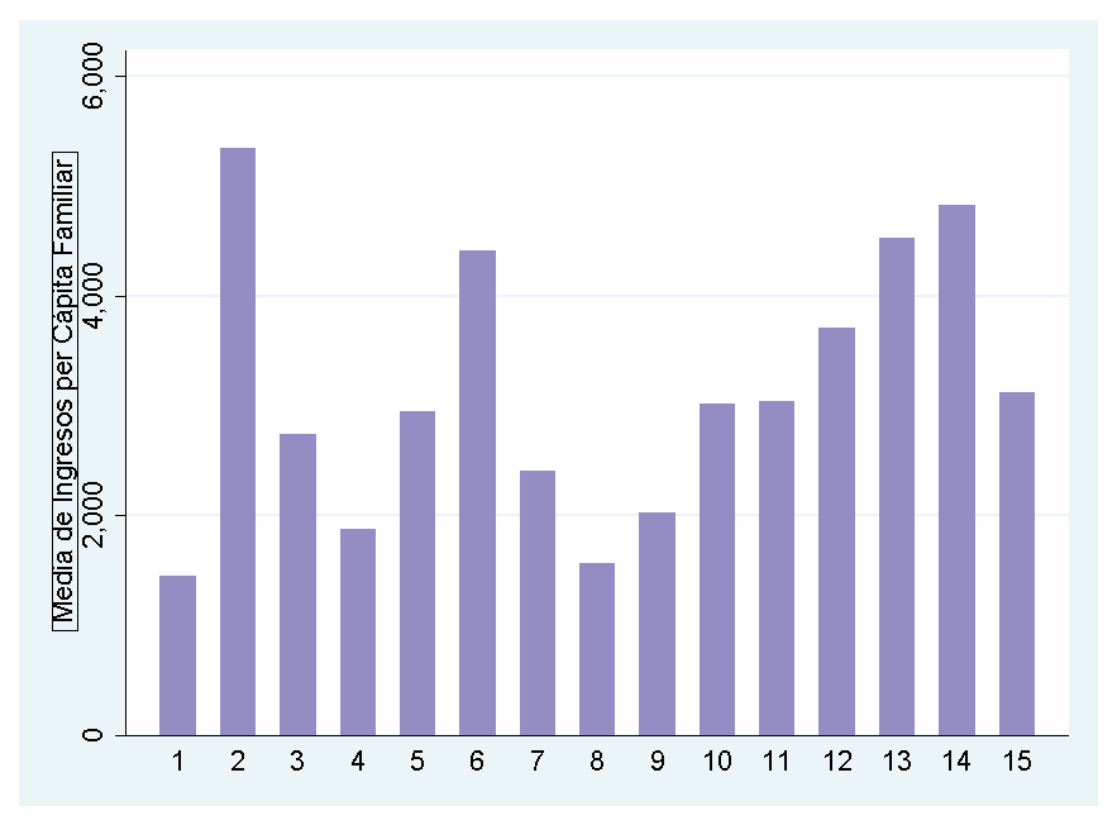

Fuente: Elaboración propia en base a EAH 2013

El gráfico 2, muestra que las diferencias económicas de las comunas se mantienen, y se profundizan cuando se observan a nivel de ingresos per cápita familiar. Las comunas «ricas», donde la media de ingreso per cápita familiar es más elevada, son la 2, 13 y 14. A estas le siguen las comunas 3, 5, 6, 11,12 y 15 de ingresos medios, y las comunas más pobres en términos de ingresos per cápita familiar de la Ciudad son las comunas 1, 4, 7, 8 y 9, que son las que se encuentran en la zona sur.

\section{Oferta de Servicios de desarrollo infantil y cuidado}

Los establecimientos de desarrollo infantil son todas las instituciones de cuidado y educación infantil vinculadas al sistema educativo formal (de gestión estatal y privada), y las instituciones no formales o vinculadas a las áreas sociales de los gobiernos provinciales, municipales, o de carácter comunitario, sostenidas por organizaciones de la sociedad civil, grupos comunitarios, gremios, sindicatos, etc. (Ministerio de Desarrollo Social y UNICEF 2012a).

La principal distinción entre estos establecimientos se vincula al hecho de que sus objetivos sean educativos o asistenciales. Los primeros pertenecen a la órbita del sistema educativo y por ello brindan servicios educativos y pedagógicos con objetivos curriculares obligatorios diseñados por el Ministerio de Educación, y llevados adelante por educadoras profesionales (según lo establecido por el art. 25 de la Ley 26206). Los establecimientos asistenciales, por su parte, se focalizan sobre todo en familias en situación de vulnerabilidad social y se orientan al fortalecimiento familiar, apoyo nutricional, contención social, con sólo algunos contenidos educativos, ya que el personal que trabaja con los niños no está obligado a tener formación docente, ni a seguir un plan curricular específico.

\footnotetext{
${ }^{5}$ Las comunas son unidades administrativas en las que se divide territorialmente la Ciudad, agrupando a los Barrios Porteños.

6 En este gráfico las comunas 1 y 7 aparecen como comunas de ingresos bajos y medios respectivamente, esta situación distorsiona el nivel de ingresos medios de la población de estas comunas. En el caso de la comuna 1 esto se debe a la presencia de villas de emergencias en el barrio de Retiro y zonas de mayor vulnerabilidad social en la zona de Constitución, con barrios como Puerto Madero y San Nicolás (zona de microcentro y de gran actividad bancaria). En el caso de la comuna 7 esto se debe a la presencia de fábricas y zonas de mayor vulnerabilidad social.
} 
A su vez, en el caso de los establecimientos asistenciales existen diferencias entre estos dependiendo de si los mismos son de gestión local o nacional, y entre servicios estatales (ya sean de gestión nacional o local) y servicios comunitarios. En el caso de los servicios educativos, estos varían dependiendo si son de gestión privada o pública pero todos están obligados a brindar los mismos contenidos mínimos impartidos por docentes matriculados.

Los servicios que se brindan en los establecimientos de desarrollo infantil de carácter asistencial se encuentran regidos por la Ley 26.233 de Promoción y Regulación de los Centros de Desarrollo Infantil Comunitarios, promulgada en el año 2007 y la Ley 27.064 de regulación y supervisión de instituciones de educación no incluidas en la enseñanza oficial, y sus decretos reglamentarios, donde se establecen estándares de calidad para el desarrollo de los establecimientos.

Por su parte, los establecimientos que se encuentran dentro de la órbita del sistema educativo formal se encuentran regidos por la Ley de Educación Nacional 26.206 (y su modificatoria de los artículos relacionados a Educación Inicial por la Ley 27.045 de 2015). Asimismo, esta ley plantea la importancia de la vinculación entre los establecimientos de tipo asistenciales y el Ministerio de Educación a fin de garantizar que se impartan contenidos educativos mínimos en este tipo de establecimientos. Cuestión que genera una tensión cuando los servicios asistenciales son utilizados como espacios de contingencia frente a la falta de bacantes en los establecimientos de enseñanza oficial. Esto implica que si bien los establecimientos de desarrollo infantil asistenciales tienen objetivos diferentes a los educativos, y se encuentran regidos por diferentes normativas, desde el Gobierno Nacional y de la Ciudad se entiende que los establecimientos de corte asistencial también son parte del plan de educación inicial que tiene por fin garantizar el derecho a la educación a todas las personas. No obstante, las diferencias en los servicios que prestan unos y otros varían notablemente.

En la última década se ha incrementado notablemente la matrícula de nivel inicial en la Ciudad, siendo el incremento en jardín maternal muy notorio en el cambio de siglo (Marzonetto, 2016). Probablemente esto esté relacionado parcialmente a que en los años de la crisis económica el deterioro de los salarios obligó a muchas mujeres a incorporarse al mercado de empleo, y con ello las necesidades de cuidado de los niños por fuera del hogar se vieron en aumento, y al crecimiento poblacional del país. Pero, si bien el incremento de la matrícula de nivel inicial a lo largo de los años a simple vista parece implicar una mejora en el acceso a los servicios educativos en la primera infancia, la evidencia nos demuestra que el incremento de la matrícula viene acompañado por un crecimiento notable de la participación de establecimientos de gestión privada, mientras la matrícula en establecimientos de gestión estatal se han mantenido prácticamente estática en la última década (ver gráfico 3).

\section{GRÁFICO 3. EVOLUCIÓN MATRÍCULA DE NIVEL INICIAL EN CABA}

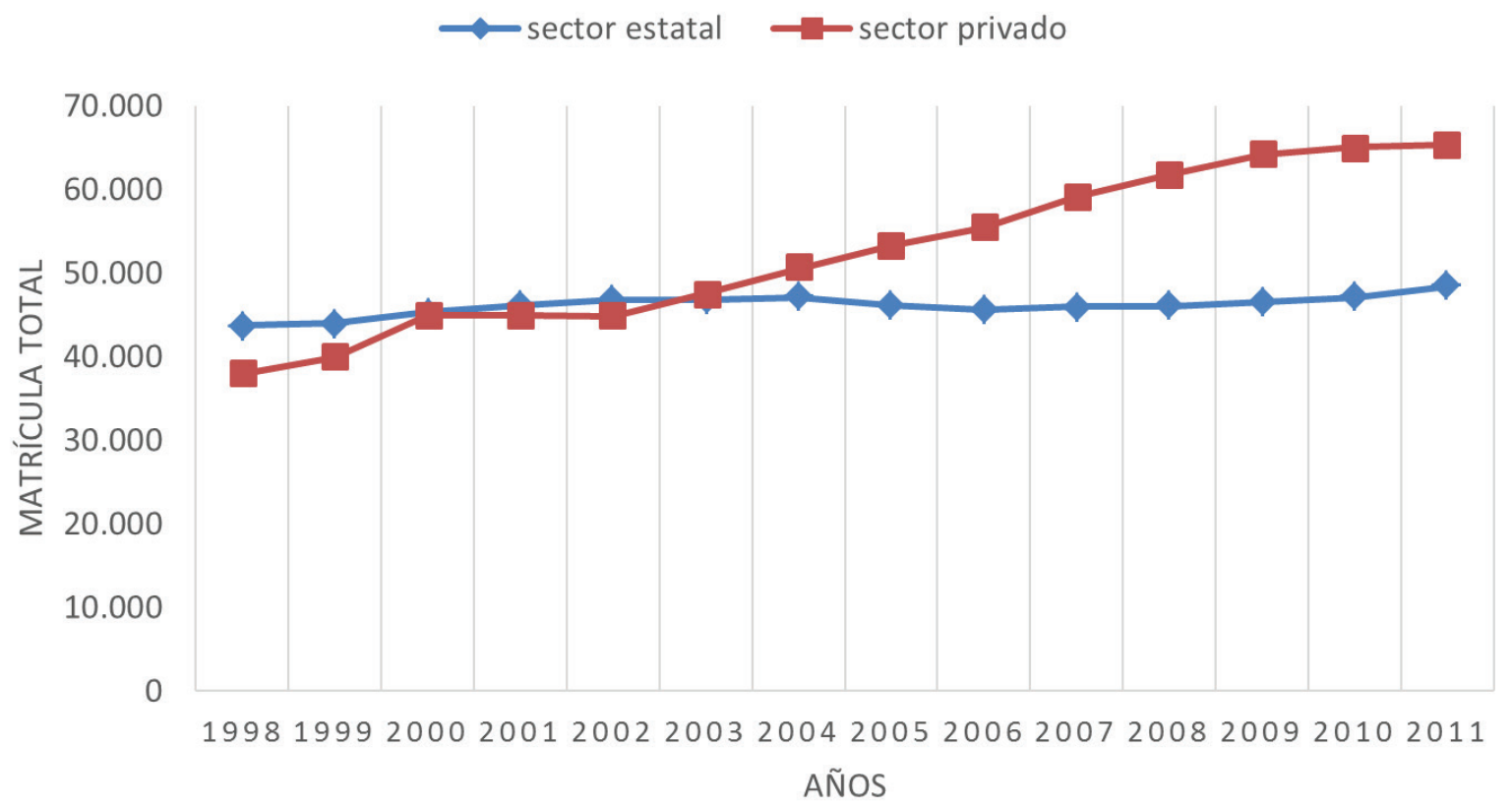

Fuente: Elaboración propia en base a Anuario Estadístico de la Ciudad de Buenos Aires, 2011 
Como muestra la tabla 1, la cobertura resulta prácticamente universal en el nivel primario para el total del país y para la CABA, con niveles superiores al 99\%. La cobertura es también elevada para la edad obligatoria dentro del nivel inicial (5 años), con una cobertura promedio del 91,4\%, y en la CABA presenta un $96,7 \%$ que supera la media nacional. A partir de allí, la cobertura de las distintas salas del nivel inicial varía sustantivamente. Para los 4 años son relativamente altas en el total del país (69,8\%), lo que podría estar explicado por la inclusión en la Ley 26.206 de la obligatoriedad del Estado de garantizar el acceso a la educación para los niños y niñas de esta edad hasta lograr su universalización. Aún con este nivel promedio para el total del país, se puede observar que la cobertura es mucho más alta en la Ciudad de Buenos (con cobertura superior al $90 \%)^{7}$.

TABLA 1. ASISTENCIA Y COBERTURA EDUCATIVA DE NIÑOS Y NIÑAS (0 A 12 AÑOS)

\begin{tabular}{cccc}
\hline Edad & Población & $\begin{array}{c}\text { Matrícula escolar total } \\
\text { (público y privado) }\end{array}$ & Cobertura \% \\
\hline $\mathbf{0}$ a $\mathbf{2}$ & 2.029 .712 & TOTAL PAIS & \\
\hline $\mathbf{3}$ & 648.071 & 78.553 & 3,9 \\
\hline $\mathbf{4}$ & 659.869 & 261.498 & 40,4 \\
\hline $\mathbf{5}$ & 676.130 & 460.653 & 69,8 \\
\hline $\mathbf{6}$ a $\mathbf{1 2}$ & 4.794 .340 & 617.709 & 91,4 \\
\hline & $\mathbf{C I U D A D}$ DE BUENOS AIRES & 98,9 \\
\hline $\mathbf{0}$ a $\mathbf{2}$ & 102.190 & 14.243 & 13,9 \\
\hline $\mathbf{3}$ & 31.572 & 23.944 & 75,8 \\
\hline $\mathbf{4}$ & 31.876 & 29.008 & 91 \\
\hline $\mathbf{5}$ & 32.338 & 31.280 & 96,7 \\
\hline $\mathbf{6}$ a $\mathbf{1 2}$ & 214.422 & & 212.647 \\
\hline
\end{tabular}

Fuente: Elaboración propia en base a datos del Censo 2010 y Relevamiento anual 2012 de la Dirección Nacional de Información y Evaluación de Calidad Educativa (DiNIECE). Ministerio de Educación de la Nación

Las diferencias en las matrículas se ven acentuadas para los niños y niñas de menor edad. Para el total del país, la cobertura educativa de los niños de 3 años apenas alcanza al $40 \%$ mientras que para la CABA es notablemente mayor, pero igualmente alarmante al estar por fuera del sistema alrededor de un $25 \%$ de los niños y niñas de esta edad. La situación se profundiza para los niños de entre 45 días y 2 años, demostrando que hay un gran porcentaje de niños y niñas de menos de 3 años que no están recibiendo cuidados por fuera de sus hogares, lo que implica que la mayor parte del cuidado de estos recae en las familias, y/o a través del mercado (contratación de trabajadoras de casas particulares).

Otra característica importante de la provisión de servicios educativos es la participación de establecimientos de gestión estatal y privada. Como se observa en la tabla 2 la matrícula escolar en primera infancia crece a medida que se acerca a la edad obligatoria de escolaridad, siendo la matrícula de jardines maternales solo el 5,6\% del total de la matrícula de nivel inicial, mientras que la de sala de 5 años es el $42,4 \%$ de la matrícula de nivel inicial total. No obstante, como advertíamos en párrafos anteriores, la situación es sensiblemente mejor para el caso de la CABA, en lo que respecta a matrícula de jardines maternales ya que representa casi el $15 \%$ de la matricula total del nivel inicial.

7 En enero de 2015 se sancionó la Ley 27045 que modifica los artículos 16,18 y 19 de la ley 26206, y ratifica la obligatoriedad de asistencia escolar para los niños y niñas a partir de los cuatro años, asimismo establece la obligación del Estado de garantizar el acceso universal a los niños y niñas de tres años de edad. Si bien se considera que la sanción de esta ley tendrá un importante impacto en la universalización de la sala de cuatro años e implicará un avance en materia educativa, debido a su reciente sanción no será posible analizar el panorama general a la luz de esta ley para los fines de este artículo. 
GAPP. Nueva Época - N.o 18, noviembre 2017 - ISSN: 1989-8991 - DOI: 10.24965/gapp.v0i18.10464 - [Págs. 43-61]

Estratificación socio-espacial y por ingresos en los servicios de desarrollo infantil y cuidado en la Ciudad de Buenos Aires

Gabriela L. Marzonetto

TABLA 2. MATRÍCULA DE ALUMNOS DE NIVEL INICIAL POR JURISDICCIÓN, POR CICLO Y TIPO DE GESTIÓN PARA EL TOTAL DEL PAÍS Y CABA, EN PORCENTAJES

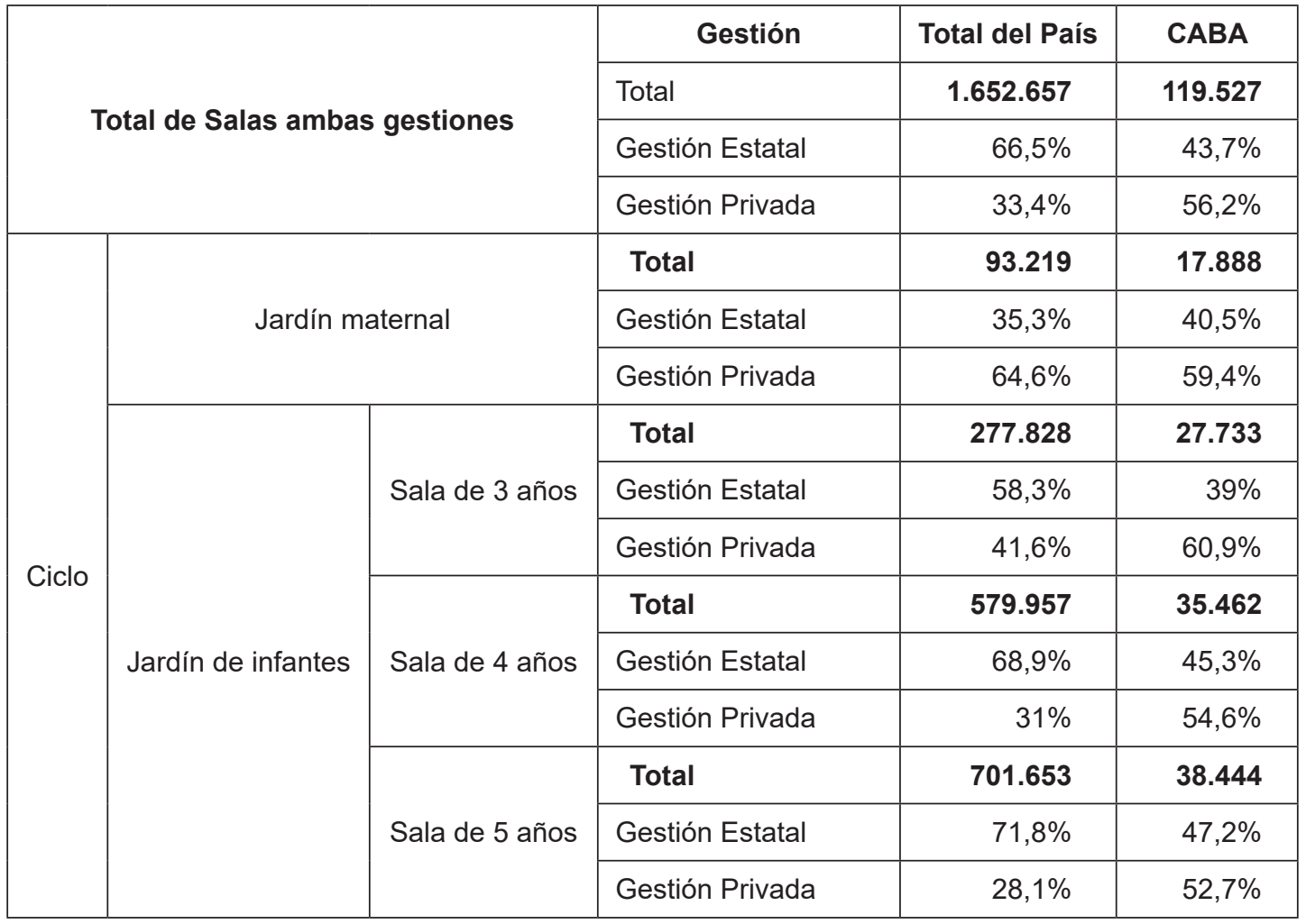

Fuente: Elaboración propia en base a datos del Relevamiento Anual 2013 de la DiNIECE. Ministerio de Educación de la Nación

Un aspecto fundamental refiere a la característica de la oferta educativa en relación a la duración de la jornada, lo que es clave toda vez que las instituciones educativas suelen ser uno de los mecanismos más habituales de conciliación entre la vida laboral y familiar (Esquivel et al., 2012; Rodriguez Enríquez y Pautassi, 2014). Según la información disponible de la Dirección Nacional de Información y Evaluación de la Calidad Educativa (DiNIECE) para el año 2013 el 22\% de los jardines maternales y el 24,1\% de los jardines de infantes de la Ciudad ofrecían servicios de doble jornada, lo que demuestra el déficit de esta oferta como mecanismo de conciliación. De todos modos, es importante resaltar que de los establecimientos que brindan servicios de doble jornada el $71,3 \%$ es de gestión estatal.

La tasa de asistencia escolar, sobre todo en los niños más pequeños, difiere marcadamente por estrato socio-económico. Adicionalmente, como analizamos a continuación, la evidencia muestra diferencias en el nivel de asistencia a espacios de cuidado por edad y por tipo de gestión de acuerdo a la comuna de residencia. Esto implica que, al menos en lo que respecta a servicios de atención en primera infancia y cuidados, existen patrones de segregación espacial que acompañan los procesos de estratificación por ingresos.

\subsection{Análisis de medias de ingresos per cápita familiares de las familias con niños que asisten a establecimientos de desarrollo infantil}

Con el propósito de analizar empíricamente la estratificación social asociada a los servicios de cuidado y desarrollo infantil en la Ciudad de Buenos Aires a continuación se analiza, en base a información recogida por Encuesta Anual de Hogares de la CABA (EAH) del año 2013, la correlación entre nivel de ingresos, la comuna de residencia y tipo de establecimiento al que se accede.

En primer lugar se realiza un análisis de 'comparación de medias' y un análisis de 'test de diferencia de medias' de ingresos per cápita familiares (IPCF) de las familias con niños que asisten a establecimientos de desarrollo infantil, por comuna y por diferencias entre servicios públicos y privados, para observar la relación entre los ingresos familiares y el tipo de servicio al que los niños asisten. En segundo lugar se realiza un 
'modelo Logit' a fin de establecer la significancia estadística en la correlación entre la asistencia a establecimientos de desarrollo infantil y los ingresos familiares y ponderar la probabilidad de concurrencia a estos establecimientos según el nivel de ingreso familiar. El mismo nos muestra la probabilidad de asistencia a establecimientos de desarrollo infantil por nivel de ingresos familiares, permitiéndonos de ese modo completar el análisis estadístico y la puesta a prueba de la hipótesis.

Si analizamos la diferencia en las medias de ingresos familiares per cápita de los niños que asisten a los establecimientos de desarrollo infantil (públicos y privados) según nivel al que asisten (tabla 3), observamos que el ingreso per cápita promedio de las familias de niños en los primeros niveles educativos es significativamente mayor, evidenciando así que la posibilidad de desfamiliarizar su cuidado aumenta junto con los ingresos familiares.

Se trabajó con dos test de medias, por un lado se realizaron 'test de diferencia de medias', que se trata de un clásico test de hipótesis que busca comparar dos poblaciones y sus muestras para comparar el comportamiento de dichas poblaciones a través de los promedios de las mismas. Por otro lado, se trabajó con observaciones simples de medias de IPCF de las familias que enviaban a sus hijos a establecimientos de desarrollo infantil (el mismo se realizó por nivel educativo, comuna de residencia y tipo de gestión de los establecimientos) a fines de conocer el nivel de IPCF de los niños que asisten a cada nivel por tipo de establecimiento. La utilización de ambos tipos de test nos permitió robustecer la puesta a prueba de la hipótesis.

Se llevaron a cabo test de diferencia de medias de dos muestras para comparar el nivel de IPCF de las familias que envían a sus hijos a establecimientos de desarrollo infantil, en relación al nivel educativo, tomando los niveles: Jardín Maternal, Sala de 3 años, Sala de 4 años y Sala de 5 años, para niños y niñas de entre 45 días y 5 años de edad. Para lograr la comparación entre medias se realizaron seis test estadísticos (tres test de comparación de diferencias de medias de los niveles sala de 3 , sala de 4 y sala de 5 con jardín maternal, dos test de comparación de diferencia de medias de los niveles sala de 4 y sala de 5 con sala de 3 , y un test para comparar las diferencias de medias de IPCF de sala de 4 y 5 ). (Ver tabla 3 ).

TABLA 3. DIFERENCIA DE MEDIA DE INGRESOS PER CÁPITA FAMILIAR DE LOS NIÑOS DE LA CABA SEgÚN NIVEL EDUCATIVO AL QUE ASISTEN. EN PESOS CORRIENTES PARA EL AÑo 2013

\begin{tabular}{|c|c|c|c|c|c|}
\hline \multicolumn{6}{|c|}{ Diferencia de Medias de IPCF por nivel de escolaridad al que asiste } \\
\hline & Media & $\begin{array}{l}\text { Error } \\
\text { estándar }\end{array}$ & $\mathbf{T}$ & Sig. (test de 2 colas) & Obs. \\
\hline \multicolumn{6}{|c|}{ Diferencia entre Jardín Maternal y ... } \\
\hline Sala de 3 & $659,23^{*}$ & 323,92 & 2,03 & 0,0428 & 285 \\
\hline Sala de 4 & $945,49^{* \star *}$ & 299,07 & 3,16 & 0,0017 & 302 \\
\hline Sala de 5 & $1.212,8^{* * *}$ & 285,67 & 4,24 & 0,0001 & 356 \\
\hline \multicolumn{6}{|c|}{ Diferencia entre Sala de 3 y ... } \\
\hline Sala de 4 & 286,25 & 239,53 & 1,19 & 0,2330 & 311 \\
\hline Sala de 5 & $553,57^{*}$ & 237,96 & 2,33 & 0,0205 & 365 \\
\hline \multicolumn{6}{|c|}{ Diferencia entre Sala de 4 y ... } \\
\hline Sala de 5 & 267,32 & 218,76 & 1,22 & 0,2225 & 382 \\
\hline
\end{tabular}

\footnotetext{
${ }^{* *} p \leq, 05 ;{ }^{* *} p \leq, 01 ;{ }^{* * *} p \leq, 001$

Fuente: Elaboración propia en base a EAH 2013
}

La información que se presenta en la tabla 3 indica que la diferencia de media de IPCF de las familias que envían a sus hijos a Jardín Maternal y a Sala de 5 es estadísticamente significativa, con un valor $p$ de 0,0001 , por lo cual podemos afirmar que se puede observar una diferencia de IPCF notable entre las familias que envían a sus hijos a jardín maternal y las que envían a sus hijos a sala de 5 años, un patrón similar se observa entre las familias que envían a sus hijos a jardín maternal y sala de 4 años, aunque las diferencias de ingresos se reducen en un $22 \%$ respecto a la brecha entre los que asisten a Jardín Maternal y Sala de 5 
años. Asimismo, la diferencia de media de IPCF entre las familias que mandan a sus hijos a jardín maternal y a sala de 3 , si bien es estadísticamente significativa, se reduce notablemente.

En relación a las diferencias de medias de IPCF de las familias cuyos hijos e hijas asisten a sala de 3 y de 4 años, la misma no es estadísticamente significativa, así como tampoco lo es la diferencia de medias de IPCF de las familias que envían a los niños a sala de 4 y 5 años. Empero, la diferencia de medias de IPCF de las familias que envían a sus hijos a sala de 3 y sala de 5 sí resulta estadísticamente significativa, con un valor $p$ de 0,02 .

La diferencia de ingresos observada en la tabla 3 evidencia que los niños que asisten a jardín maternal en la CABA pertenecen a familias cuyo IPCF promedio es significativamente más alto que los niños que asisten a los otros niveles educativos ${ }^{8}$. Cuestión que puede estar explicada por el elevado costo de los jardines maternales privados y la escasa oferta pública, ya que sólo hay 15 jardines maternales de gestión pública en todo el territorio de la Ciudad.

De estos datos se desprende también que a medida que se acerca la edad obligatoria de asistencia escolar los ingresos familiares de los niños que asisten a los establecimientos decrece. Esto podría ser explicado en parte por el impacto de la obligatoriedad escolar a partir de los 5 años que genera que, a medida que se aproxima la edad de ingreso obligatorio, entren compulsivamente al sistema educativo niños y niñas de familias de menores ingresos y a su vez, que de todos los niveles educativos del nivel inicial, la sala de 5 años es la que cuenta con mayor cantidad de establecimientos de gestión pública ${ }^{9}$.

La evidencia sugiere que hay una relación entre el nivel de ingresos familiares y el tipo de establecimiento al que asisten los niños; cuestión que influye sobre las opciones de las familias de resolver el cuidado de sus hijos por fuera del hogar.

Al analizar la evidencia observando las diferencias por tipo de comuna (entre comunas de ingresos altos, medios y bajos), surge que las diferencias en los ingresos familiares en relación al nivel educativo tienden a mantenerse, y que los ingresos varían ampliamente entre las comunas de mayores ingresos y de menores ingresos de la ciudad, como se observa en el gráfico 4. No obstante, encontramos algunos datos contra-intuitivos. Por ejemplo se observa que los niños de las comunas de ingresos bajos asisten más al jardín maternal que los niños de las comunas de ingresos medios. Esto puede estar explicado en parte por el sesgo generado en la interpretación de la pregunta «¿qué nivel está cursando actualmente?» de la EAH, que no distingue entre establecimientos de desarrollo infantil de tipo educativo y asistencial, y en parte por la caída del número de observaciones cuando trabajamos por nivel educativo y comuna de residencia. Sin embargo, los niños de comunas de ingresos altos y medios asisten más al resto de los niveles que los niños de las comunas consideradas pobres.

GRÁFICO 4. MEDIAS DE INGRESO PER CÁPITA FAMILIAR POR TIPO DE COMUNA, SEGÚN NIVEL EDUCATIVO AL QUE ASISTEN LOS NIÑOS DE LA CABA. INGRESOS EN PESOS CORRIENTES AÑO 2013

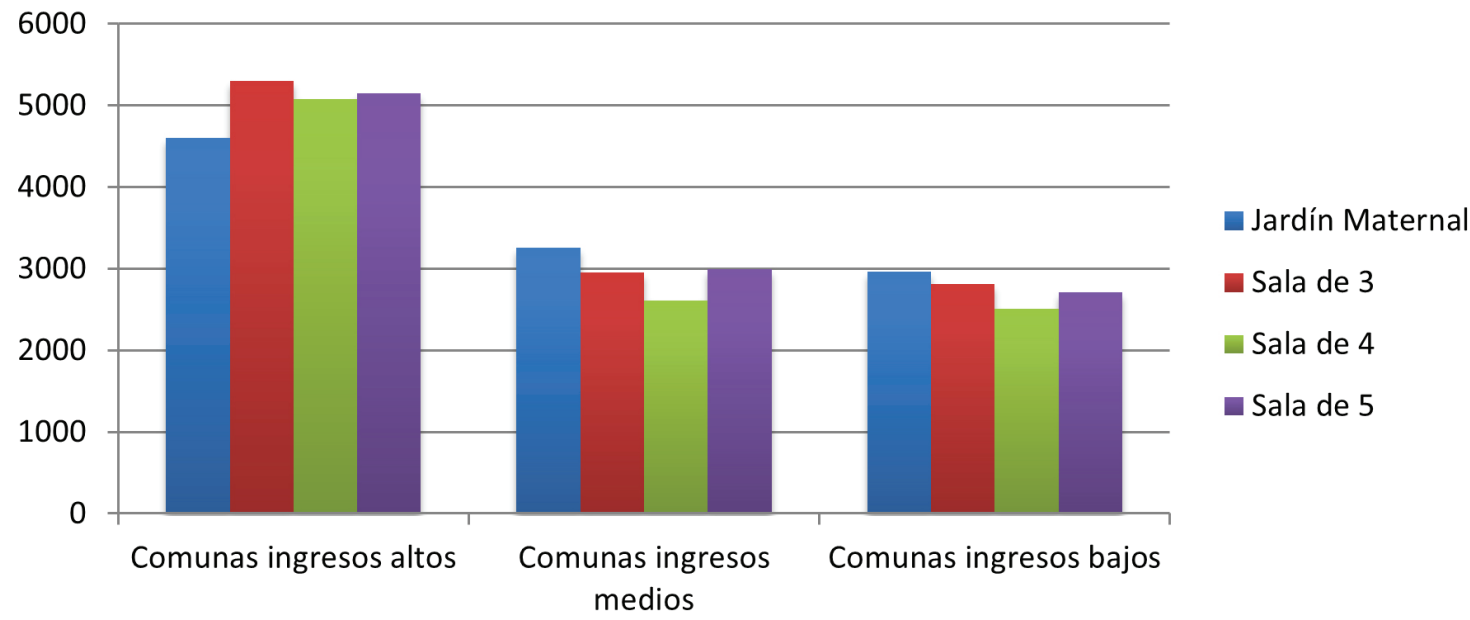

Fuente: Elaboración propia en base a EAH 2013

\footnotetext{
8 22,2\% en comparación con sala de 3; 34,7\% en comparación con sala de 4 y 48,4\% en comparación con sala de 5 .

9 Hasta el 2014 la edad de ingreso obligatorio era de 5 años.
} 
En el caso de la asistencia a Jardín Maternal de los niños de las comunas de ingresos altos, puede considerarse la posibilidad de que esta situación de menor participación en relación con la participación en salas de 3 años, sea explicada por la opción de no enviar a los niños pequeños al jardín en los primeros años de vida y resolver el cuidado de los mismos a través de contratación de niñeras o por medio de la familia (Faur, 2014; Ministerio de Desarrollo Social y UNICEF, 2012). Asimismo, la participación en Jardín Maternal de los niños de las comunas de ingresos altos es mayor que en el resto de las comunas.

En el gráfico 5 observamos la distribución de los ingresos de las familias por nivel educativo. Se advierte, como mencionamos más arriba, que el ingreso medio más elevado lo tienen las familias que envían a sus hijos al jardín maternal, seguido de la sala de 3 , luego sala de 4 y, por último, la sala de 5 . A su vez, también encontramos que los ingresos más elevados de cada grupo también siguen el mismo patrón.

GRÁFICO 5. DIAGRAMA DE CAJAS DE COMPARACIÓN DE MEDIAS DE IPCF POR NIVEL EDUCATIVO ${ }^{10}$

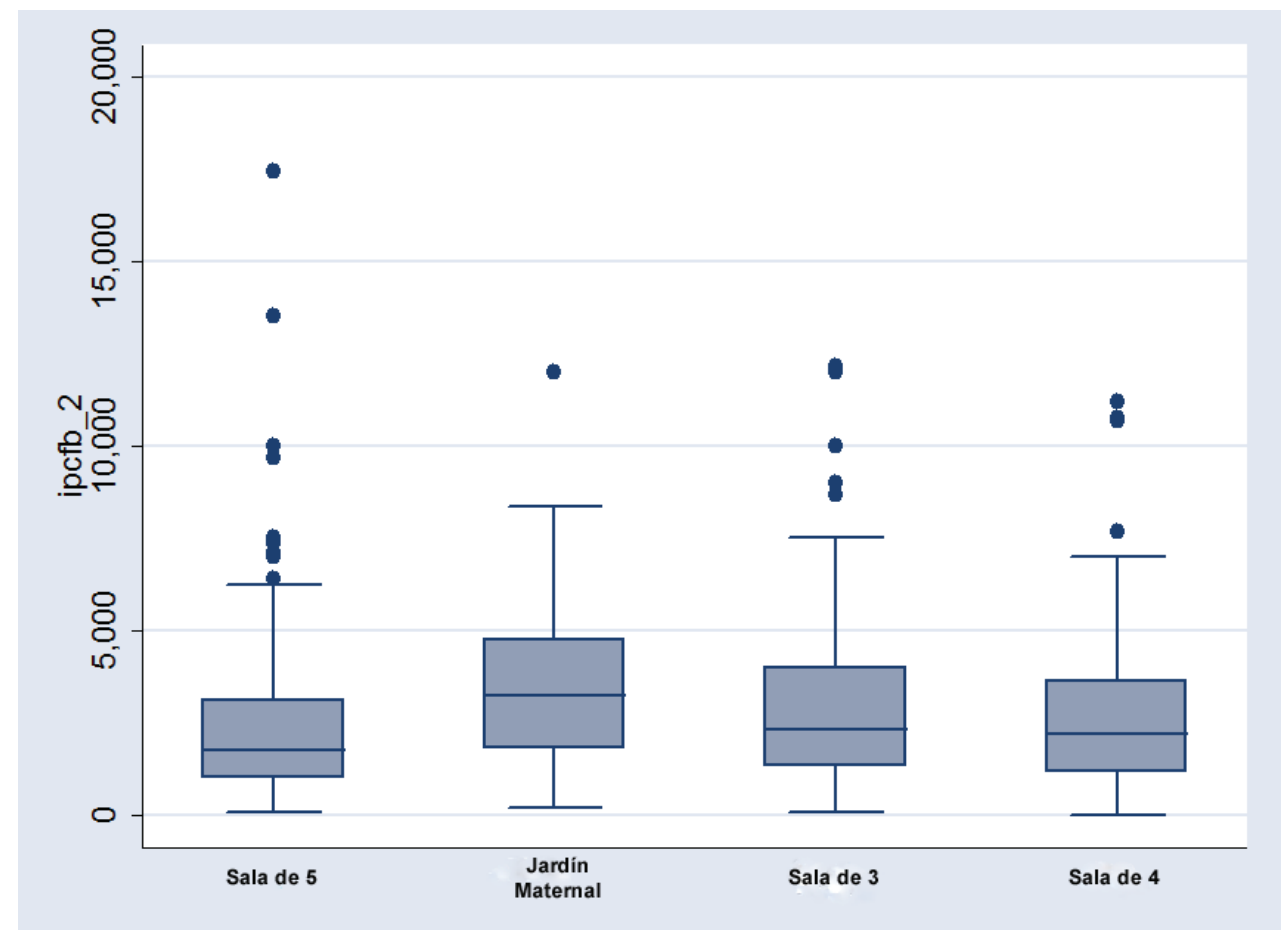

Fuente: elaboración propia en base a EAH 2013

Cuando analizamos ingresos medios por tipo de establecimiento y nivel educativo, parece sostenerse el patrón por el cual el tipo de establecimiento al que asisten los niños varía en relación a los ingresos medios de las familias, siendo menores en establecimientos públicos, mayores en establecimientos privados laicos e intermedios en privados religiosos (ver gráfico 6). A su vez, los ingresos familiares medios son mayores en las familias con niños en Jardín Maternal en todos los tipos de establecimiento, redundado en lo que señalábamos al comienzo respecto a que son los niños pertenecientes a familias con mayores ingresos las que más concurren a establecimientos de desarrollo infantil desde temprana edad (ver tabla 4).

La comuna de procedencia también limita las opciones sobre el tipo de establecimiento de desarrollo infantil, no obstante es posible que los niños concurran a establecimientos alejados de sus hogares. Por ello, para poder evidenciar la correlación entre ingreso y tipo de establecimiento debemos utilizar información a nivel de los hogares. Como muestra el gráfico 7 , los ingresos familiares promedio en las familias que envían a sus niños a establecimientos privados son mayores a los de las familias que concurren a establecimientos públicos, y estos ingresos a su vez son en general mayores en las comunas de ingresos altos.

10 El orden de presentación de los diagramas de cajas del gráfico 5 se debe al el modo en que fueron codificadas las variables en la EAH 2013. 
GRÁFICO 6. DIAGRAMA DE CAJAS DE COMPARACIONES DE MEDIAS DE IPCF DE LAS FAMILIAS POR NIVEL EDUCATIVO Y TIPO DE ESTABLECIMIENTO (TODAS LAS COMUNAS) ${ }^{11}$

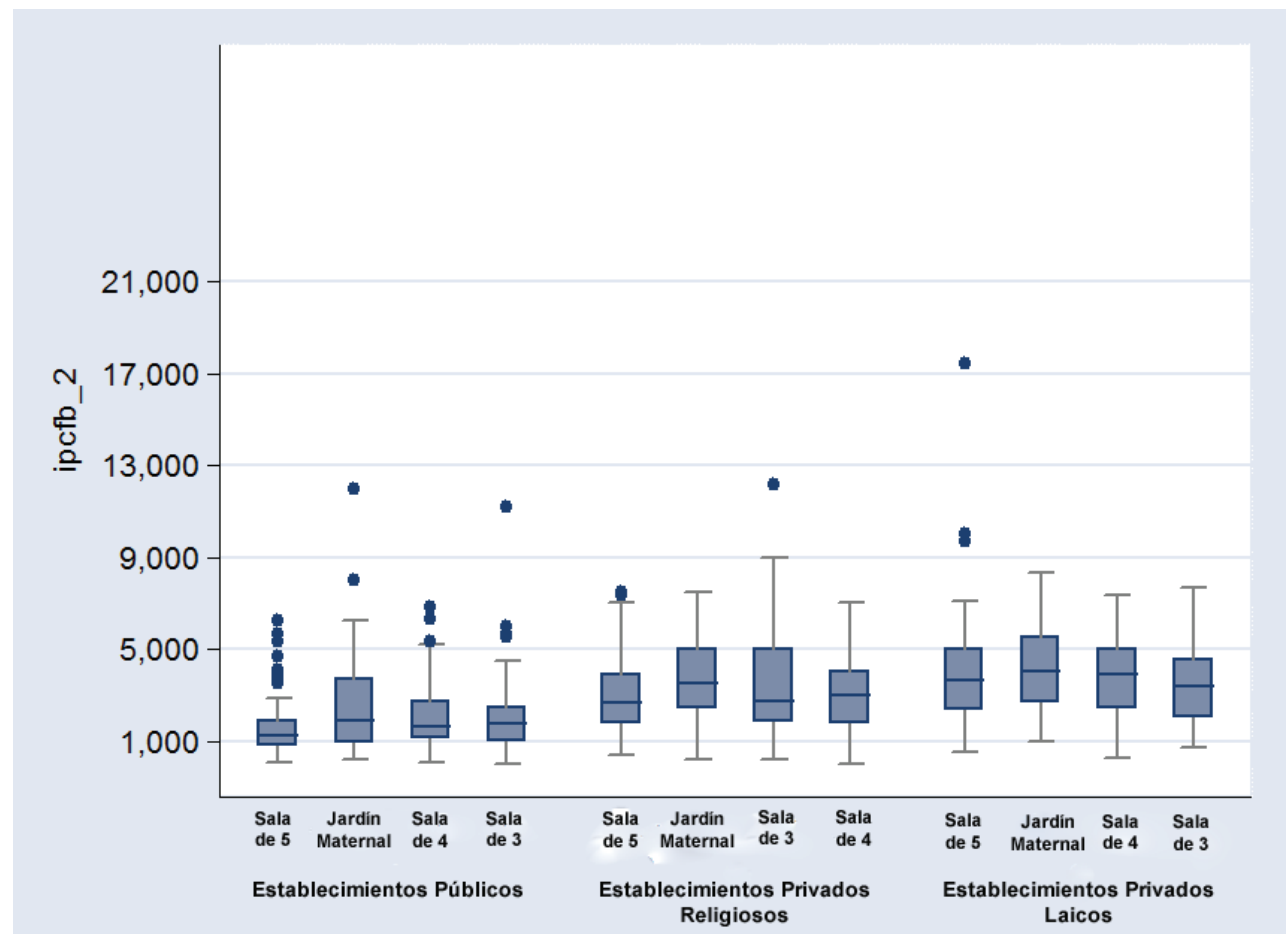

Fuente: elaboración propia en base a EAH 2013

GRÁFICO 7. MEDIAS DE IPCF DE LAS FAMILIAS POR TIPO DE COMUNA Y TIPO DE ESTABLECIMIENTO (TODOS LOS NIVELES)

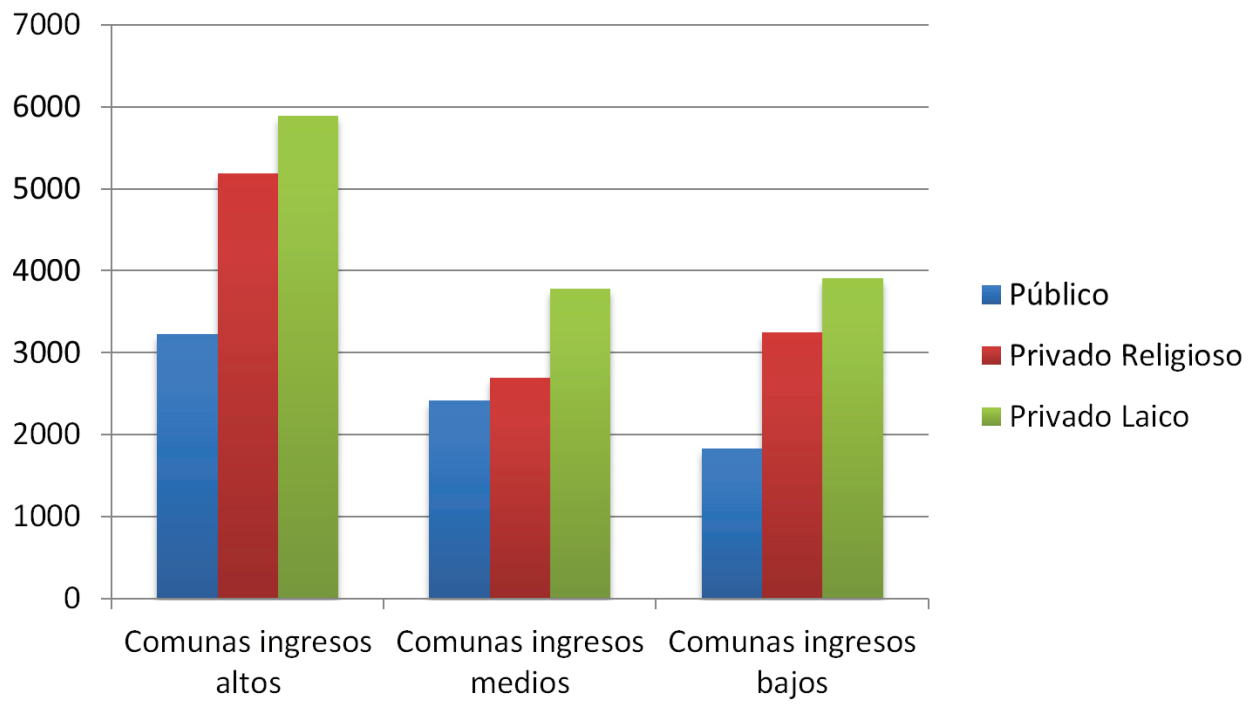

Fuente: elaboración propia en base a EAH 2013

Otra distinción relevante se da entre establecimientos privados religiosos y establecimientos privados laicos. Los primeros suelen ser subvencionados por el Estado, por lo que son en general más baratos que

11 El orden de presentación de los diagramas de cajas del gráfico 6 se debe al el modo en que fueron codificadas las variables en la EAH 2013. 
GAPP. Nueva Época - N. 18, noviembre 2017 - ISSN: 1989-8991 - DOI: 10.24965/gapp.v0i18.10464 - [Págs. 43-61]

Estratificación socio-espacial y por ingresos en los servicios de desarrollo infantil y cuidado en la Ciudad de Buenos Aires

Gabriela L. Marzonetto

los laicos. Por ello, la distinción entre unos y otros es relevante, sobre todo para identificar sectores medios que optan por salir del sistema público (o no consiguen bancos en este) y no tienen recursos suficientes para acceder a cualquier establecimiento privado ${ }^{12}$. La evidencia disponible sostiene esta distinción ya que es clara la correlación entre tipo de comuna, nivel de ingresos de las familias y tipo de establecimiento: las familias que concurren a establecimientos públicos tienen ingresos ligeramente inferiores a aquellas que concurren a establecimientos privados religiosos y estas, a su vez, tienen ingresos ligeramente inferiores a las que concurren a establecimientos privados laicos (ver tabla 4).

TABLA 4. COMPARACIÓN DE MEDIAS DE IPCF DE LAS FAMILIAS POR NIVEL EDUCATIVO Y TIPO DE ESTABLECIMIENTO (TODAS LAS COMUNAS). EN PESOS CORRIENTES DEL AÑO 2013

\begin{tabular}{llc}
\hline Nivel educativo & Tipo de establecimiento & IPCF promedio simple \\
\hline Jardín Maternal & Público & $2.494,7$ \\
\hline & Privado Religioso & $3.636,7$ \\
\hline Sala de 3 & Privado Laico & $4.717,7$ \\
\hline & Público & $2.142,8$ \\
\hline & Privado Religioso & $3.843,2$ \\
\hline Sala de 4 & Privado Laico & $4.149,2$ \\
\hline & Público & $2.026,3$ \\
\hline & Privado Religioso & $2.926,3$ \\
\hline Sala de 5 & Privado Laico & $3.945,7$ \\
\hline & Público & $1.562,4$ \\
\hline & Privado Religioso & $3.363,4$ \\
\hline
\end{tabular}

Fuente: elaboración propia en base a EAH 2013

Al realizar un test de diferencia de medias para observar las diferencias de IPCF de las familias por tipo de establecimiento educativo al que envían a los niños, observamos que la diferencia presentada en el tabla 5 se mantiene, con una significancia estadística alta (con un valor $p$ de 0,001 ) para la diferencia entre todos los casos (tabla 5). En este caso se realizaron 12 test estadísticos: test diferencia de medias de IPCF entre establecimientos públicos y privados religiosos; entre establecimientos públicos y privados laicos; entre establecimientos privados religiosos y privados laicos, todos estos para los cuatro niveles educativos (jardín maternal, sala de 3 , sala de 4 y sala de 5 ).

TABLA 5. DIFERENCIAS DE MEDIAS DE IPCF DE LAS FAMILIAS POR TIPO DE ESTABLECIMIENTO Y NIVEL EDUCATIVO (TODAS LAS COMUNAS)

\begin{tabular}{|c|c|c|c|c|c|c|}
\hline \multicolumn{7}{|c|}{ Diferencia de Medias de IPCF por tipo de establecimiento al que asiste } \\
\hline Tipo de establecimiento & Nivel & Media & $\begin{array}{c}\text { Error } \\
\text { estándar }\end{array}$ & $\mathbf{T}$ & $\begin{array}{l}\text { Sig. (test } \\
\text { de } 2 \\
\text { colas) }\end{array}$ & Obs. \\
\hline \multicolumn{7}{|c|}{ Diferencia entre Público y ... } \\
\hline \multirow[t]{2}{*}{ Privado Religioso } & Jardín Maternal & $2.756,86^{* \star *}$ & 235,09 & 11,72 & 0,0001 & 78 \\
\hline & Sala de 3 & $2.624,18^{\star \star *}$ & 201,45 & 13,03 & 0,0001 & 116 \\
\hline
\end{tabular}

12 Esta apreciación debe ser completada con estudios que analicen en qué medida la religiosidad de las familias es un importante factor que influye en la preferencia entre colegios religiosos-subvencionados y colegios laicos (públicos o privados); lamentablemente no contamos con datos para poder ponderar esta cuestión. 
GAPP. Nueva Época - N.1, noviembre 2017 - ISSN: 1989-8991 - DOI: 10.24965/gapp.v0i18.10464 - [Págs. 43-61]

Estratificación socio-espacial y por ingresos en los servicios de desarrollo infantil y cuidado en la Ciudad de Buenos Aires

Gabriela L. Marzonetto

\begin{tabular}{|c|c|c|c|c|c|c|}
\hline & Sala de 4 & $2.322,08^{\star \star \star}$ & 148,34 & 15,65 & 0,0001 & 130 \\
\hline & Sala de 5 & $2.003,28^{* * *}$ & 128,78 & 15,55 & 0,0001 & 183 \\
\hline \multirow[t]{4}{*}{ Privado Laico } & Jardín Maternal & $3.599,52^{\star \star *}$ & 306,01 & 11,76 & 0,0001 & 119 \\
\hline & Sala de 3 & $2.676,22^{* * *}$ & 177,28 & 15,09 & 0,0001 & 113 \\
\hline & Sala de 4 & $2.553,20^{\star * *}$ & 193,31 & 13,21 & 0,0001 & 117 \\
\hline & Sala de 5 & $2.125,95^{\star * *}$ & 161,55 & 13,16 & 0,0001 & 169 \\
\hline \multicolumn{7}{|c|}{ Diferencia entre Privado Religioso y ... } \\
\hline \multirow[t]{4}{*}{ Privado Laico } & Jardín Maternal & $4.441,71^{* * *}$ & 401,22 & 11,07 & 0,0001 & 79 \\
\hline & Sala de 3 & $3.972,15^{\star \star *}$ & 328,57 & 12,08 & 0,0001 & 79 \\
\hline & Sala de 4 & $3.336,17^{* * *}$ & 229,84 & 14,51 & 0,0001 & 81 \\
\hline & Sala de 5 & $3.834,5^{* * *}$ & 299,31 & 12,81 & 0,001 & 84 \\
\hline
\end{tabular}

${ }^{* * *} p \leq, 001$

Fuente: elaboración propia en base a EAH 2013

Observamos también que las diferencias de IPCF en relación al tipo de establecimiento varían por nivel educativo, siendo en la comparación entre los tres casos mayores la diferencia de IPCF para jardín maternal que para sala de 5 años.

\subsection{Modelo Logit sobre el impacto del nivel de ingresos per cápita familiar en la probabilidad de asistencia a establecimientos de desarrollo infantil}

Con el objeto de verificar si la correlación entre la asistencia a establecimientos de desarrollo infantil y el nivel de ingresos familiares tiene significancia estadística se llevó a cabo un modelo Logit. A continuación describiremos las variables utilizadas en este modelo.

\section{Variable dependiente}

La variable dependiente es la asistencia a establecimiento de desarrollo infantil.

En base a la pregunta: ¿qué nivel está cursando actualmente? (variable «e6») aplicada a niños de entre 45 días y 5 años, se construyó una variable dicotómica («e6a») que marca la condición de estar o no concurriendo a un establecimiento educativo para todos estos niveles.

\section{Variable Independiente}

Ingreso per Cápita Familiar: La principal variable independiente es el ingreso per cápita familiar (variable «ipcfb_2») de las familias que envían a los niños a establecimientos educativos. Esta variable, como hemos visto a lo largo del trabajo, reviste de importancia para analizar la estratificación de los servicios de cuidado y desarrollo infantil en la CABA.

\section{Variables de control}

El modelo se controló por dos variables ${ }^{13}$ : Tipo de Hogar y Sexo.

Tipo de Hogar: se utilizó esta variable (tipoho) para observar si existían diferencias en la asistencia de los niños a los establecimientos de desarrollo infantil según la composición familiar. Para poder utilizar esta variable como variable de control, se tuvo que integrar las bases de datos de la EAH individual con la EAH

13 Las variables de control posibles a utilizar para este estudio se encuentran condicionadas a las variables incorporadas en la $\mathrm{EAH}$. Por ello, en base a la información disponible se procuró utilizar las variables más relevantes en términos teóricos. 
GAPP. Nueva Época - N.1 18, noviembre 2017 - ISSN: 1989-8991 - DOI: 10.24965/gapp.v0i18.10464 - [Págs. 43-61]

Estratificación socio-espacial y por ingresos en los servicios de desarrollo infantil y cuidado en la Ciudad de Buenos Aires

Gabriela L. Marzonetto

hogares y así construir una única base de datos que integrara información a nivel individual con información sobre los hogares.

Sexo: en el modelo se buscaba observar si había diferencia en la asistencia a establecimientos educativos en base al sexo de niños y niñas de entre 45 días y 5 años que asisten a estos establecimientos.

Debido a que nuestra variable dependiente es categórica (asistencia a establecimiento de desarrollo infantil) y nuestra principal variable independiente (IPCF) es continua, se optó por un modelo de regresión logística que nos permitiera observar la significancia estadística de la relación entre estas variables y realizar un cálculo de probabilidades de asistencia a establecimientos en base al IPCF.

Es importante tomar en consideración una serie de aspectos en relación a la evidencia. El análisis estadístico se hizo en base a la EAH 2013 (última disponible al momento de la investigación). Debido a que el cuestionario tiene por objetivo recabar diversa información que permita obtener un panorama general de la situación socioeconómica y las condiciones de vida de las personas que viven en la ciudad de Buenos Aires, las preguntas referidas a escolaridad son escasas y si bien permitieron observar la participación de los niños a los distintos niveles de escolaridad, no permitieron identificar entre servicios de tipo educativo o asistencial -cuestión que queda sujeta a la interpretación de la persona entrevistada pudiendo generar sesgos en la información-. En esta línea, cuando se realizó el modelo logit la idea principal era utilizar como variables de control el nivel educativo de la madre y de ambos padres, y si los jefes de hogar de los niños que asisten a establecimientos están empleados en el sector formal o informal, pero por el diseño del cuestionario fue imposible trabajar con estas variables, cuestión que hubiera resultado ser un aporte valioso para el análisis estadístico.

\subsubsection{Modelo logit}

El modelo muestra que el coeficiente de la relación entre las variables «asistencia a establecimientos de desarrollo infantil» e «IPCF» es positivo, lo que indica que el aumento en el ingreso familiar aumenta la probabilidad de que los niños y niñas concurran a un establecimiento de desarrollo infantil. A su vez, este efecto es estadísticamente significativo, con un valor $p$ de 0,001 (tabla 6).

TABLA 6. MODELO LOGIT. NIVEL DE INGRESOS PER CÁPITA FAMILIAR Y LA PROBABILIDAD DE ASISTENCIA A ESTABLECIMIENTOS DE DESARROLLO INFANTIL

\begin{tabular}{l|c}
\hline \multicolumn{1}{c}{ Modelo Logit. Asistencia a Establecimientos de desarrollo infantil } \\
\hline \multirow{2}{*}{ IPCF (ipcfb_2) } & $0,0018^{* * *}$ \\
\cline { 2 - 2 } & $(0,001)$ \\
\hline \multirow{2}{*}{ Comuna } & $0,0311^{*}$ \\
\hline \multirow{2}{*}{ Tipo de Hogar (tipoho) } & $(0,015)$ \\
\hline \multirow{2}{*}{ Sexo } & 0,0159 \\
\cline { 2 - 2 } & $(0,035)$ \\
\hline \multirow{2}{*}{ Constante } & 0,0922 \\
\cline { 2 - 2 } & $(0,120)$ \\
\hline
\end{tabular}

Pseudo R_2: 0,030

Número de observaciones: 1.154

Errores Estándar entre paréntesis. * $p \leq, 05 ;{ }^{* *} p \leq, 01 ; * * * p \leq, 001$

Fuente: Elaboración propia en base a EAH 2013

Para calcular el efecto del ingreso sobre la probabilidad de asistir a un establecimiento de desarrollo infantil se utilizan los logits de las probabilidades binomiales, como establece la siguiente fórmula: 


$$
\operatorname{logit}\left(\rho_{\mathrm{i}}\right)=\ln \left(\frac{\rho_{\mathrm{i}}}{1-\rho_{\mathrm{i}}}\right)=\beta_{0}+\beta_{1} X_{1 \mathrm{i}}
$$

Donde $\rho_{\mathrm{i}}$ es igual a la suma de los productos entre los coeficientes de la variable independiente y la constante y los valores asignados a la variable independiente y la constante para la simulación. En la tabla 7 se muestran los valores utilizados:

TABLA 7. SUMA DE LOS PRODUCTOS DE LOS COEFICIENTES DE LA VARIABLE IPCF Y LA CONSTANTE PARA LA SIMULACIÓN DEL CÁLCULO DE PROBABILIDADES

\begin{tabular}{lrrr}
\hline & Coeficientes & Valores & Sumaproducto \\
\hline IPCF & 0,0001818 & 3.300 & $-0,1639043$ \\
\hline Constante & $-0,76384430$ & 1,00 & \\
\hline & & & \\
\hline IPCF & 0,0001818 & 12.000 & 3,78115570 \\
\hline Constante & $-0,76384430$ & 1,00 & \\
\hline
\end{tabular}

Fuente: Elaboración propia en base a EAH 2013

El valor de para un ingreso familiar estimado de $3.300 \$$ es de $-0,1639043$, por ello el cálculo de la probabilidad de concurrencia a un establecimiento de desarrollo infantil es:

$$
\operatorname{logit}\left(\rho_{\mathrm{i}}\right)=\ln \left(\frac{-0,1639043}{1-(-0,1639043)}\right)=0,45911541
$$

Y el valor de para un ingreso familiar estimado de $12.000 \$$ es de 1,41775570 , por ello el cálculo de la probabilidad de concurrencia a un establecimiento de desarrollo infantil es:

$$
\operatorname{logit}\left(\rho_{\mathrm{i}}\right)=\ln \left(\frac{1,41775570}{1-(1,41775570)}\right)=0,80498634
$$

En base al modelo se puede estimar entonces que si una familia en 2013 tenía un ingreso per cápita familiar de 3.300\$ la probabilidad de que su hijo de entre 45 días y 5 años asistiera a un establecimiento de desarrollo infantil era de un $46 \%$. Si en cambio la familia tenía un ingreso per cápita familiar de $12.000 \$$, la probabilidad de asistencia era del $80 \%$.

\section{DISCUSIÓN}

Podemos afirmar que a mayor nivel de ingresos familiares mayores son las probabilidades de asistencia a establecimientos de desarrollo infantil en la primera infancia, lo cual corrobora nuestra hipótesis principal.

En relación a las variables de control observamos que ni el tipo de hogar al que pertenecen los niños, ni el sexo de los mismos tiene efectos estadísticamente significativos en la asistencia a establecimientos de desarrollo infantil. Un aspecto ya señalado que la evidencia corrobora, es que los niños que asisten a jardines maternales provienen de familias con mayores IPCF promedio que los niños que asisten a los demás niveles (sala de 3,4 y 5). También se observa que a medida que se acerca la edad de escolaridad obligatoria (sala de 4 y 5) la media de IPCF promedio de las familias disminuye notablemente. Esto da indicios de que los niños de menores ingresos ven retrasado su ingreso a establecimientos de desarrollo infantil, y con ello se retrasan sus oportunidades de recibir estímulos pedagógicos desde la primera infancia, lo que coadyuva a la reproducción de las desigualdades sociales.

Por último, el modelo logit demostró que la correlación entre los ingresos familiares y la probabilidad de que los niños asistan a establecimientos de desarrollo infantil es estadísticamente significativa y que el «tamaño del efecto» es sustantivamente relevante. 
Cuando se observa que de los niños y niñas que asisten al nivel inicial en la Ciudad, más de la mitad lo hace en establecimientos privados, se evidencian por un lado, la falta de servicios educativos de gestión estatal para este tramo etario, y por otro lado, la desigualdad de oportunidades de acceso a estos servicios dependiendo del nivel de ingresos familiares.

El análisis estadístico en base a la asistencia de niños y niñas a los servicios de cuidado y primera infancia permite corroborar la hipótesis al demostrar que los servicios de cuidado y desarrollo infantil funcionan como mecanismos de reproducción de la estratificación social en la CABA. La evidencia fue contundente:

- Los niños que asisten a jardín maternal pertenecen a familias de ingresos más altos que los que asisten a los demás niveles, observándose que los niños de familias de menores ingresos aumentan su asistencia a medida que se acerca la edad de escolaridad obligatoria.

- Los niños de comunas más pobres asisten más a establecimientos de gestión pública mientras los niños de comunas de ingresos altos asisten más a establecimientos de gestión privada.

- En todos los grupos de comunas (comunas de ingresos altos, comunas de ingresos medios, comunas de ingresos bajos) se presenta la tendencia a que los niños de familias de mayores ingresos concurran a establecimientos privados laicos, los niños de familias de ingresos medios asistan a establecimientos privados religiosos y los niños de familias de menores ingresos asistan a establecimientos de gestión estatal.

- La relación entre la asistencia a establecimientos de desarrollo infantil y el nivel de ingresos familiares es estadísticamente significativa, lo que implica que las probabilidades de asistencia a un establecimiento de desarrollo infantil aumentan a medida que aumentan los ingresos familiares.

Todo esto da indicios de que los niños de menores ingresos ven retrasado su ingreso a establecimientos de desarrollo infantil, y con ello se retrasan sus oportunidades de recibir estímulos pedagógicos desde la primera infancia, lo que genera una brecha en las oportunidades a futuro en los mismos. Esto, concomitantemente, repercute en la capacidad de las familias de resolver el problema del cuidado de los niños pequeños lo que a su vez limita las posibilidades de las mujeres de liberar parte de su tiempo, que podría permitirles participar en el mercado de empleo -que a su vez contribuiría a mejorar las condiciones económicas de las familias de menores recursos-. Ante esta evidencia, consideramos que es necesario replantearnos la actual organización social del cuidado y con ello el diseño de los servicios que desde el Estado se proveen (y se regulan, en el caso de los ofrecidos en el mercado) que tienen por fin garantizar el desarrollo integral infantil y la desfamiliarización -aunque parcial- del cuidado de niños y niñas.

Si bien el debate a nivel regional sobre la importancia de fortalecer y expandir este tipo de políticas ha penetrado en las agendas públicas nacionales, siendo los casos más emblemáticos el uruguayo, el chileno y el costarricense (Blofield y Martínez Franzoni, 2014), en Argentina que paradojamente tiene un Estado de bienestar históricamente activo y una tradición en materia de servicios educativos públicos desde el nivel inicial, el problema del cuidado de los niños pequeños se ha incorporado tibiamente en el repertorio de la agenda pública local. Esto conlleva una serie de problemáticas no sólo en el plano familiar individual, donde las mujeres son las que realizan mayor parte de las tareas de cuidado y los niños ven afectado su desempeño escolar, sino que también en el plano social, puesto que no brindar las mismas oportunidades de base a todos los niños -tanto por problemas de acceso o por calidad de los servicios destinados a estos-, refuerza y reproduce la herencia social, cuestión que profundiza el problema de la estratificación y segmentación social a futuro. Por esto, y en consonancia con las lecciones de Esping-Andersen (2010) y Pallier (2011) en base al desarrollo del tema en los países industrializados, se considera que para lograr un reparto equitativo y viable de las cargas que pesan en nuestras sociedades es necesario redoblar los esfuerzos en materia de inversión en primera infancia.

\section{REFERENCIAS BIBLIOGRÁFICAS}

ADDABBO, T. y DI TOMMASO, M. L. (2014). Children well-being and family characteristics in Italy. International Association for Feminist economist.

BARNETT, W. S. (2013). Long-term effects of Early Childhood Programs on Cognitive and School outcomes, 5(3), 25-50. DOI: $10.2307 / 1602366$

BATTHYÁNY, K. (2015). Los tiempos del bienestar social. Género, trabajo no remunerado y cuidados en Uruguay. 
GAPP. Nueva Época - N. 18, noviembre 2017 - ISSN: 1989-8991 - DOI: 10.24965/gapp.v0i18.10464 - [Págs. 43-61]

Estratificación socio-espacial y por ingresos en los servicios de desarrollo infantil y cuidado en la Ciudad de Buenos Aires

Gabriela L. Marzonetto

BLOFIELD, M. y MARTÍNEZ FRANZONI, J. (2014). Una década de cambios en las relaciones entre vida familiar y laboral: mayor corresponsabilidad estatal; incipiente corresponsabilidad paterna. Cepal.

BUDLENDER, D. (2008). The Statistical Evidence on Care and Non-Care Work across Six Countries (4).

ENGLAND, P., BUDIG, M. y FOLBRE, N. (2002). "Wages of Virtue: The Relative Pay of Care Work". Social Problems, 49(4), 455-473. DOI: 10.1525/sp.2002.49.4.455.

ESPING-ANDERSEN, G. (1993). Los tres mundos del Estado de Bienestar (Pensament). Valencia: Institucio Alfons el Magnanim.

ESPING-ANDERSEN, G. (2008). "The three political economies of the welfare state". Canadian Review of Sociology/ Revue canadienne de sociologie, 26(1), 10-36. DOI: 10.1111/j.1755-618X.1989.tb00411.x.

ESPING-ANDERSEN, G. (2010). The incomplete revolution. Adapting to women's new roles (2nd ed.). Cambridge: Polity Press.

ESPING-ANDERSEN, G. y PALIER, B. (2011). Los tres grandes retos del Estado de bienestar. Barcelona: Editorial Ariel.

ESQUIVEL, V. (2009). "Uso del tiempo en la Ciudad de Buenos Aires". En Seminario virtual de Especialización en género, economía y desarrollo en el contexto de la crisis (pág. 70). Buenos Aires: Universidad Nacional de General Sarmiento.

ESQUIVEL, V. (2012). "EI cuidado infantil en las familias. Un análisis en base a la Encuesta de Uso del Tiempo de la Ciudad de Buenos Aires”. En V. ESQUIVEL, E. FAUR y E. JELIN (eds.), Las lógicas del cuidado infantil. Entre las familias, el Estado y el Mercado (págs. 73-106). Buenos Aires: IDES.

ESQUIVEL, V., FAUR, E., y JELIN, E. (2012). Las lógicas del cuidado infantil. Entre las familias, el estado y el mercado. Buenos Aires: IDES.

FAUR, E. (2014). El cuidado infantil en el Siglo XXI. Mujeres malabaristas en una sociedad desigual. Buenos Aires, Argentina: Siglo Veintiuno editores.

FELFE, C., NOLLENBERGER, N., y RODRÍGUEZ-PLANA, N. (2012). Can't Buy Mommy's Love? Universal Childcare and Children's Long-Term Cognitive Development.

HASKINS, R. (1989). "Beyond metaphor: The efficacy of early childhood education". American Psycologist, 44, $274-82$. DOI: 10.1037/0003-066x.44.2.274.

LOCURTO, C. (1991). Beyond IQ in preschool programs? Intelligence, 7, 73-104

MARZONETTO, G. (2016). Educación Inicial y servicios asistenciales para la primera infancia en la Ciudad de Buenos Aires: derechos universales, servicios estratificados (Documentos de Trabajo núm. 93). Buenos Aires.

MINISTERIO DE DESARROLLO SOCIAL y UNICEF. (2012). Encuesta sobre condiciones de vida de niñez y adolescencia 2011, 2012.

PAKULSKI, J. (2006). "Social Stratification". En B. TURNER (ed.), The Cambridge Dictionary of Sociology. New York: Cambridge University Press.

PAUTASSI, L. y ZIBECCHI, C. (2010). "La provisión de cuidado y la superación de la pobreza infantil. Programas de transferencia condicionadas en Argentina y el papel de las organizaciones sociales y comunitarias". Políticas Sociales, núm. 159. Santiago de Chile.

RAZAVI, S. (2007). The Political and Social Economy of Care in a Development Context (núm. 3). Geneva.

RAZAVI, S. (2011). "Rethinking Care in a Development Context: An Introduction". Development and Change, 42(4), 873-903. DOI: 10.1111/j.1467-7660.2011.01722.x.

RITZER, G. y MICHAEL, R. (2011). The concise encyclopedia of sociology. West Sussex, UK: Wiley-Blackwell ed. DOI: $10.1002 / 9781444392654$.

RODRÍGUEZ ENRÍQUEZ, C. (2015). "El trabajo de cuidado no remunerado en Argentina: un análisis desde la evidencia del Módulo de Trabajo no Remunerado”. Políticas Públicas y derecho al cuidado núm. 2. Buenos Aires, Argentina.

RODRÍGUEZ ENRÍQUEZ, C. y Marzonetto, G. (2015). "El trabajo de cuidado remunerado: Estudio de las condiciones de empleo en la educación básica y en el trabajo en casas particulares”. Políticas Públicas y derecho al cuidado núm. 2. Buenos Aires.

RODRÍGUEZ ENRÍQUEZ, C. y PAUTASSI, L. (2014). La organización social del cuidado de niños y niñas. Elementos para la construcción de una agenda de cuidados en Argentina. (Rodriguez Enriquez y Pautassi, eds.). Buenos Aires, Argentina: Equipo Latinoamericano de Justicia y Género: ELA.

SOJO, A. (2011). De la evanescencia a la mira: El cuidado como eje de políticas y de actores en América Latina. Santiago de Chile.

STAAB, S. y GERHARD, R. (2011). "Putting Two and Two Together? Early Childhood Education, Mothers' Employment and Care Service Expansion in Chile and Mexico". Development and Change, 42(4), 1079-1107. DOI: $10.1111 / \mathrm{j} .1467-7660.2011 .01720 . x$.

SUNKEL, G. (2006). "Políticas Familiares y Regímenes de Bienestar en América Latina", en Reunión de Expertos en Gestión y Financiamiento de las Políticas que Afectan a las Familias (pág. 12). Santiago de Chile: CEPAL.

UNICEF. (2014). Building Better Brains: New frontiers in Early Childhood development.

ZASLOW, M. (1991). "Variation in child care quality and its implications for children". Journal of Social issues, 47(2), 125-39. DOI: 10.1111/j.1540-4560.1991.tb00291.x. 